\title{
SISTEMAS DE CAPTACIÓN DE AGUA LLUVIA PARA LA PRODUCCIÓN AGROPECUARIA SOSTENIBLE
}

RUBÉN MEDINA CARRILLO

Investigación y Extensión Universidad Estatal a Distancia, Guanacaste, Costa Rica rmedina@uned.ac.cr

WAGNER PEÑA CORDERO

Escuela de Ciencias Exactas y Naturales Universidad Estatal a Distancia, Costa Rica wpena@uned.ac.cr

MARÍA FÉLIX OBANDO BRICEÑO

Universidad Estatal a Distancia, Guanacaste, Costa Rica mobando@uned.ac.cr

\section{RESUMEN}

El presente artículo analiza la factibilidad técnica y financiera al implementar un sistema de capacitación de agua lluvia o reservorio en beneficio de la asociación de agricultores orgánicos de Cerro Verde de Nicoya (antes Cerro Negro), y utilizar esta estructura para la producción agropecuaria sostenible. El estudio se realizó en el segundo semestre de 2010 y primer trimestre de 2011. El Reservorio - Sistema de captación de agua lluvia instalado fue de suma importancia para la producción, ya que permitió a los productores almacenar agua que se utilizó en la agricultura en época seca, y en la época lluviosa se implementó la producción de Tilapias aumentando los niveles de producción. Los sistemas de captación de agua lluvia son milenarios, en la actualidad países como Brasil, Israel, México, Estados Unidos, India, China, entre otros, utilizan sistemas de captación de agua lluvia para la producción. En Costa Rica el tema de cosecha de agua solo se ha empezado a publicar recientemente a raíz del proyecto de cosecha de agua de las universidades públicas. La rentabilidad financiera al instalar el sistema de captación de agua lluvia o reservorio se determinó mediante estudios económicos que consideraran la inversión inicial, costos de producción, gastos financieros y gastos administrativos. Se analizaron métodos financieros que se utilizan para evaluación de proyectos como lo son el Valor Actual Neto (VAN), la Tasa Interna de Retorno (TIR) y la razón beneficio/costo (R. b/c) obteniendo resultados financieramente viables en los escenarios planteados.

PALABRAS CLAVE: TECNOLOGÍA, SCALL, RENTABILIDAD FINANCIERA, PRODUCCIÓN AGROPECUARIA, MERCADO DE TILAPIAS.

\section{ABSTRACT}

This article analyzes the technical and financial feasibility of implementing a training system for a rainwater reservoir for the association of organic farmers in Cerro Verde of Nicoya (former Black Mountain), and use this framework for sustainable agricultural production. Most of the study was done in the second half of 2010 and the first quarter of 2011. The reservoirs installed were of great importance to the production and allowed farmers to store water which was used in both seasons. During the dry season the water was used for agriculture. During the rainy season it was used to increase production of tilapia. Systems of rainwater harvesting have been used since ancient times. Currently, systems are being used for rainwater harvesting and production in countries such as Brazil, Israel, Mexico, United States, India, and China. Costa Rica has expertise in the subject, but this knowledge has not been shared until recently. Now it can be replicated in other regions for sustainable food production. Financial 
returns to install the reservoir were determined by economic studies which considered the initial investment, production costs, financial costs and administrative expenses. The financial methods used to evaluate the projects included the Net Present Value (NPV), the internal rate of return (IRR) and benefit / cost ratio $(R \mathrm{~b} / \mathrm{c})$. The results indicate that it was financially profitable to install the system of rainwater harvesting.

KEYWORDS: TECHNOLOGY, SCALL, FINANCIAL PROFITABILITY, AGRICULTURAL PRODUCTION, TILAPIA MARKET.

\section{INTRODUCCIÓN}

En Guanacaste (provincia de Costa Rica) al igual que en otras partes del mundo resulta cada vez más importante desarrollar una gestión adecuada del agua, acción que está directamente relacionada con el desarrollo humano. El agua en la actualidad es una mercancía con un incalculable valor a nivel mundial y su escasez provoca pérdidas en la producción como sucedió en 2010:

En la región Chorotega (Guanacaste) se perdieron alrededor de 1.200 hectáreas de arroz y se afectaron unas 5.000 hectáreas de caña de azúcar y grandes extensiones de pasto. Según el informe preliminar al que tuvo acceso el periódico La Nación, en la misma región también se vieron afectadas "unas 2.000 hectáreas de maíz, 122 hectáreas de sandía y áreas menores de chile dulce, tomate, frijol y papaya. En total, las pérdidas ascendieron a $\$ 6$ millones ( $\$ 3.169$ millones) solo en esa región del Pacífico nacional (La Nación, 12 de abril 2010).

Irónicamente, a esta situación, Guanacaste es una de las provincias de Costa Rica donde más llueve con un promedio de precipitación de 1500- 2000 ( $\mathrm{mm} / a n ̃ o)$ pero de forma espaciada en los meses que van de diciembre a abril, hay escasez del recurso provocando una gran disminución en la producción agropecuaria y pérdidas millonarias en el sector que a su vez inciden en el aumento de la pobreza y desempleo en la región.

Otro escenario se muestra en los meses que van de mayo a noviembre, donde hay gran abundancia del recurso agua por las excesivas lluvias que azotan la provincia, que en ocasiones provocan inundaciones y al final este valioso recurso es depositado en el mar por los ríos de la región.

A raíz de esta problemática, las universidades públicas de Costa Rica desarrollan un proyecto que analiza, investiga e implementa 4 sistemas de captación de agua de lluvia para ser utilizados en la producción, en el país el tema de la cosecha de agua no ha sido documentada ni publicada con anterioridad al proyecto. Este artículo muestra el estudio realizado en uno de los sistemas de captación de agua lluvia que se instalaron en beneficio de la asociación de agricultores orgánicos de Cerro Verde; por lo tanto, determinar la viabilidad técnica y económica al instalar este sistema de capacitación de agua Iluvia en la provincia es de suma importancia, ya que la cosecha de agua se plantea como una alternativa de conservación y aprovechamiento en armonía con el ambiente, que permite el desarrollo de la agricultura especialmente en hortalizas y la producción piscícola.

\section{METODOLOGÍA}

\section{Recolección de la información}

El estudio se realizó en el segundo semestre de 2010 y primer semestre de 2011, la información para desarrollarlo fue obtenida del proyecto de reservorios de la Escuela de Ciencias Exactas de la Universidad Estatal a Distancia (UNED), dirigido por la Cátedra del Suelo, además información suministrada por la Universidad Técnica de Costa Rica en materia de Piscicultura. 
Descripción de las áreas de estudio

El área de estudio comprende la Región Chorotega de Costa Rica, la muestra de la investigación fue realizada con los datos obtenidos al instalar, desarrollar y producir con el sistema de captación de agua lluvia (en adelante SCALL) implementado en la asociación de agricultores orgánicos de Cerro Verde de Nicoya.

\section{Análisis de la información}

y supuestos utilizados

La información fue tabulada y procesada en Microsoft Excel 20100. Para el análisis de los escenarios, se plantearon una serie de supuestos, basados en información recolectada de la realidad nacional.

Se utilizó un periodo de análisis a 10 años, el cual es el estándar en análisis de rentabilidad similar. El análisis monetario se realizó en dólares americanos para tratar de minimizar el efecto de la inflación.

Se utilizó una tasa comparativa en dólares (USD) de la TIR de 1,11\%, así como la tasa de la carga financiera de 7,96\% en dólares, basada en información brindada por el Banco Central de Costa Rica (20-05-2010).

\section{COSECHA DE AGUA LLUVIA PARA LA PRODUCCIÓN SOSTENIBLE}

La cosecha de agua de lluvia puede entenderse como una forma de producir agua (Cubero, 1996).

La captación de agua lluvia es milenaria, se tiene conocimiento que el ser humano ha utilizado SCALL desde hace 5 mil años, en esa época se desarrolló la irrigación a pequeña escala para minimizar el efecto de la sequía.

En Costa Rica, no existía experiencia documentada sobre sistemas de captación de agua lluvia con anterioridad al proyecto. El presente artículo analiza la factibilidad financiera y técnica al invertir en un SCALL o Reservorio en Cerro Verde de Nicoya, la mayor parte de la información de este estudio es obtenida de la Escuela de Ciencias Exactas de la UNED, del proyecto "Fortalecimiento de la seguridad alimentaria mediante el desarrollo y fomento de reservorios artificiales de aguas precipitadas, para la utilización en la agricultura sostenible, en la región chorotega de Costa Rica" financiado con fondos de Regionalización del CONARE.

\section{Inversión Inicial del SCALL}

El Reservorio o SCALL que presenta este artículo posee una capacidad de almacenamiento de $340 \mathrm{~m}^{3}$ de agua, el material de revestimiento utilizado es de la más alta calidad para este tipo de estructuras (está revestido con geotextil y geomembrana), el análisis de la inversión contempla movimientos de tierra con maquinaria, materiales de revestimiento, materiales de riego, costo de producción del cultivo de tilapia y otros costos relacionados con la producción de hortalizas, pero por tratarse de un análisis de un proyecto de agricultores los cuales poseen su parcela no se incluye el costo del terreno para desarrollar el SCALL y la producción de hortalizas.

En el tabla 1 y Figura 1, se muestra de forma resumida la inversión del proyecto, el capital de trabajo fue calculado con el método del periodo de desfase el cual calcula la inversión en capital de trabajo como la cantidad de recursos necesarios para financiar los costos de operación desde que se inician los desembolsos y hasta que se recuperan. Para ello, toma el costo promedio diario y lo multiplica por el número de días estimados de desfase, en el caso de este proyecto el periodo de desfase es de 90 días. 
TABLA 1

RESUMEN DE LA INVERSIÓN DEL PROYECTO SISTEMAS DE CAPTACIÓN DE AGUA LLUVIA PARA PRODUCCIÓN AGROPECUARIA SOSTENIBLE EN CERRO VERDE DE NICOYA

\begin{tabular}{|l|c|}
\hline \multicolumn{1}{|c|}{ Costos de operación } & USD \\
\hline Infraestructura & $\$ 4670$ \\
\hline Equipos de producción & $\$ 800$ \\
\hline Capital de trabajo & $\$ 939,32$ \\
\hline Activo nominal o intangibles & $\$ 1000$ \\
\hline TOTAL DE LA INVERSIÓN & $\$ 7499,32$ \\
\hline
\end{tabular}

Fuente: elaboración propia.

FIGURA 1

RESUMEN DE LA INVERSIÓN

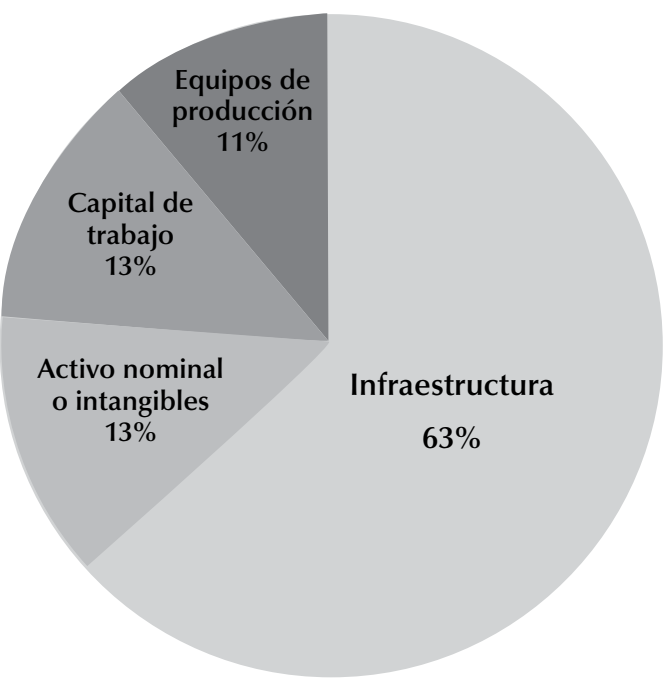

Fuente: elaboración propia.

\section{Infraestructura}

En cuanto a la Infraestructura utilizada para el montaje del sistema de riego y el reservorio, los costos de esta se presentan en la tabla 2.
TABLA 2

\section{INVERSIÓN EN INFRAESTRUCTURA DEL SISTEMA DE RIEGO Y REVESTIMIENTO DEL RESERVORIO EN USD Y POR METROS CUADRADOS}

\begin{tabular}{|l|l|}
\hline \multicolumn{1}{|c|}{} & $\begin{array}{c}\text { Costo USD } \\
\text { por m2 }\end{array}$ \\
\hline Reservorio o SCALL con geotextil y geomembrana & $\$ 12,2$ \\
\hline Sistema de Riego con goteo y micro aspersión & $\$ 0,55$ \\
\hline
\end{tabular}

\section{Movimientos de Tierra}

Se requirió maquinaria pesada para adecuar el área de implementación del reservorio, en la tabla 3 se presentan los costos.

\section{COSTOS DEL MOVIMIENTO DE TIERRAS}

\begin{tabular}{|c|c|c|c|}
\hline $\begin{array}{c}\text { Cantidad } \\
\text { de horas }\end{array}$ & Descripción & Precio unitario & Total \\
\hline 18 & Trabajos Back hoe & $\$ 36$ & $\$ 648$ \\
\hline
\end{tabular}

\section{Materiales de revestimiento e instalación}

En cuanto a la instalación del reservorio se requirió geomembrana y geotextil. El costo de estos materiales y su instalación se detallan en la Tabla 4.

TABLA 4

\section{COSTOS DE LA INSTALACIÓN DE GEOMEMBRANAY GEOTEXTIL}

\begin{tabular}{|l|l|l|l|}
\hline \multicolumn{1}{|c|}{ Material } & Cantidad & $\begin{array}{c}\text { Precio } \\
\text { unitario }\end{array}$ & \multicolumn{1}{|c|}{ Total } \\
\hline Geomembrana HDPE 1.0MM & $345 \mathrm{~m}^{2}$ & $\$ 3,908$ & $\$ 1348,26$ \\
\hline Geotextil no tejido 205 $\mathrm{gr} / \mathrm{m}^{2}$ & $345 \mathrm{~m}^{2}$ & $\$ 1,19,00$ & $\$ 410,55$ \\
\hline $\begin{array}{l}\text { Instalación geomebrana } \\
\text { y geotextil }\end{array}$ & $345 \mathrm{~m}^{2}$ & $\$ 4,04$ & $\$ 1393,8$ \\
\hline Sub total & & & $\$ 3152,61$ \\
\hline Impuesto de ventas 13\% & & & $\$ 409,83$ \\
\hline Total & & & $\$ 3562,44$ \\
\hline
\end{tabular}

Fuente: elaboración propia. 


\section{Sistemas de Riego}

Un buen sistema de riego es de gran importancia para maximizar el recurso hídrico durante la época seca, en el siguiente análisis se ha contemplado la instalación y compra de un sistema de riego que incluye riego por micro aspersión y por goteo para un lote de $240 \mathrm{~m}^{2}$, que incluye un sistema de tubería que va desde el SCALL o reservorio hasta la zona de cultivo llevando el agua por medio de la gravedad.

A continuación, se describen estos dos sistemas de riego para comprender su importancia dentro de este sistema.

\section{Riego por goteo}

En el riego por goteo el agua se aplica gota a gota a la planta, es una de las técnicas más importantes para la agricultura por maximizar el recurso agua. El riego por goteo se adapta perfectamente al sistema de captación de agua lluvia utilizado y al tipo de cultivo en estudio como lo fue la plantación de cebolla.

\section{Riego por aspersión}

El riego por aspersión es el sistema que hace llegar el agua a las plantas mediante tuberías y unos pulverizados llamados aspersores, de esta forma riega la superficie que se requiere.

\section{Producción con el sistema de captación de agua lluvia o reservorio}

Durante la época lluviosa el SCALL o Reservorio se utilizó para la producción de peces con la especie conocida como Oreochromis niloticus (Tilapia Gris) y durante la época seca se utilizó para la producción agrícola (cultivo de cebolla) de esta manera se pretende dar un uso óptimo del recurso.
Generalidades del cultivo

de tilapia en el reservorio

La tilapia es una especie que se adapta fácilmente a las condiciones climatológicas en diversos cuerpos de agua.

En el caso de la producción de tilapia en los reservorios o SCALL no solo es un incremento en el factor económico, sino que además esta especie es un control biológico de los mosquitos como por ejemplo el Aedes aegypti, que se reproduce en el agua acumulada y produce la enfermedad del Dengue. Al ser las Tilapias una especie omnívora se alimentará de cualquier larva que se produzca en el reservorio. Además, otra de las ventajas de producir tilapia en los reservorios es que se adaptan con facilidad a los diferentes tipos de alimento, tanto los producidos naturalmente como los alimentos artificiales (derivados de subproductos agrícolas).

En el caso particular de la Asociación de Agricultores Orgánicos de Cerro Verde, produjeron Tilapia Orgánica. Según el análisis técnico realizado el reservorio o SCALL tiene capacidad para producir 2000 tilapias y se recomendó la siembra de la especie Oreochromis niloticus (Tilapia Gris).

En el mes de agosto de 2010 se realizó la producción de 2000 tilapias con un promedio de 80 gramos cada una y vendidas en el mercado en el mes de Febrero de 2011 con un peso estimado de 850 gramos cada una. Es importante mencionar que la Asociación de Agricultores Orgánicos de Cerro Verde posee muy buenos puntos de comercialización y su segmento de mercado son los extranjeros que visitan Guanacaste; según los datos de ventas de la asociación, hay más demanda que oferta en todos sus productos, por lo tanto, la comercialización de este producto fue factible. De esta información se parte del supuesto que todo lo producido en este periodo como en periodos futuros será vendido a un precio promedio del mercado nacional de tilapia. 


\section{Producción agrícola utilizando el reservorio o SCALL para incrementar el ciclo de producción}

Con el agua recolectada del SCALL o reservorio y una vez comercializadas las tilapias, se pretende incrementar la producción agrícola en la época seca demostrando que almacenar agua es una opción viable que permite asegurar los requerimientos hídricos para la producción agrícola. Esta pretende ser una medida de adaptación a la problemática que, a través de la historia, ha enfrentado la provincia Guanacasteca donde la sequía amenaza año con año, provocando la perdida millonaria en cultivos como hortalizas, caña, frijoles, arroz y la ganadería, entre otros.

Requerimientos hídricos de los Cultivos

Los cultivos requieren diferentes necesidades hídricas, las cuales se estiman con el uso consuntivo ( $U c$, en $\mathrm{mm}$ ), que se determina de la siguiente manera:

Uc $=$ ETo. Kc.

\section{$\mathrm{ETo}=$ Evapotranspiración potencial $(\mathrm{mm})$ \\ $\mathrm{Kc}=$ Coeficiente de cultivo.}

La tabla 5 muestra el requerimiento hídrico de cultivos en Costa Rica

TABLA 5

\section{COEFICIENTE DE CULTIVOS EN COSTA RICA}

\begin{tabular}{|l|l|l|l|}
\hline \multicolumn{1}{|c|}{ Cultivo } & Kc global & \multicolumn{1}{c|}{ Cultivo } & Kc global \\
\hline Aguacate & 0,85 & Papa & 0,75 \\
\hline Arroz & 1,2 & Papaya & 0,8 \\
\hline Café & 0,95 & Pepino & 0,9 \\
\hline Caña de azúcar & 0,9 & Piña & 0,5 \\
\hline Cebolla & 1 & Plátano & 0,85 \\
\hline Chile & 0,6 & Remolacha & 0,75 \\
\hline Frijol & 0,7 & Repollo & 1,1 \\
\hline
\end{tabular}

\begin{tabular}{|l|l|l|l|}
\hline Maíz & 0,85 & Sandía & 0,6 \\
\hline Mango & 0,8 & Tiquizque & 0,5 \\
\hline Zanahoria & 0,6 & Tomate & 0,7 \\
\hline Melón & 0,6 & Yuca & 0,5 \\
\hline Naranja & 0,7 & Chayote & 0,9 \\
\hline
\end{tabular}

Fuente: Boletín № 54 de la FA0, 1990.

En la tabla 6 se muestra el ciclo del cultivo de productos agrícolas en Costa Rica.

TABLA 6

CICLO DEL CULTIVO EN COSTA RICA

\begin{tabular}{|l|l|l|l|}
\hline \multicolumn{1}{|c|}{ Cultivo } & $\begin{array}{c}\text { Ciclo del } \\
\text { cultivo días }\end{array}$ & \multicolumn{1}{|c|}{ Cultivo } & $\begin{array}{c}\text { Ciclo del } \\
\text { cultivo días }\end{array}$ \\
\hline Guayaba & Anual & Maíz & 105 \\
\hline Mango & Anual & Tomate & 120 \\
\hline Café & Anual & Papa & 120 \\
\hline Ayote & Anual & Pepino & 90 \\
\hline Naranja & Anual & Piña & 240 \\
\hline Sandía & 75 & Repollo & 120 \\
\hline Caña de azúcar & Anual & Arroz & 120 \\
\hline Melón & 90 & Papaya & 180 \\
\hline Remolacha & 90 & Cebolla & 105 \\
\hline Frijol & 100 & Tiquizque & 300 \\
\hline Chile & 105 & Yuca & 300 \\
\hline Zanahoria & 120 & Plátano & Anual \\
\hline Elote & 75 & Chayote & Anual \\
\hline & & & \\
\hline
\end{tabular}

Fuente Valverde (2007).

Para el análisis del proyecto se utilizó el cultivo de cebolla por requerir un ciclo corto de producción y facilidades de comercialización. Además es un cultivo muy producido en la zona y los agricultores tienen mucha experiencia en su cultivo. 
TABLA 7

\section{PRECIO PROMEDIO MENSUAL DE LA CEBOLLA SECA EN LOS DIFERENTES MERCADOS EN COSTA RICA EN 2010 (EN COLONES/K)}

\begin{tabular}{|c|c|c|c|c|}
\hline MES & EN FINCA & CENADA & BORBON & FERIAS \\
\hline ene-10 & 360 & 463 & 644 & 560 \\
\hline feb-10 & 356 & 475 & 683 & 606 \\
\hline mar-10 & 418 & 555 & 723 & 649 \\
\hline abr-10 & 464 & 638 & 820 & 729 \\
\hline may-10 & 644 & 838 & 1038 & 938 \\
\hline jun-10 & 713 & 1038 & 1240 & 1155 \\
\hline jul-10 & 755 & 990 & 1212 & 1112 \\
\hline ago-10 & 675 & 775 & 1015 & 908 \\
\hline sep-10 & 352 & 420 & 632 & 538 \\
\hline
\end{tabular}

Fuente: Consejo Nacional de Producción (2010), servicio de información e inteligencia de mercados tomado el 09 de octubre de 2010.

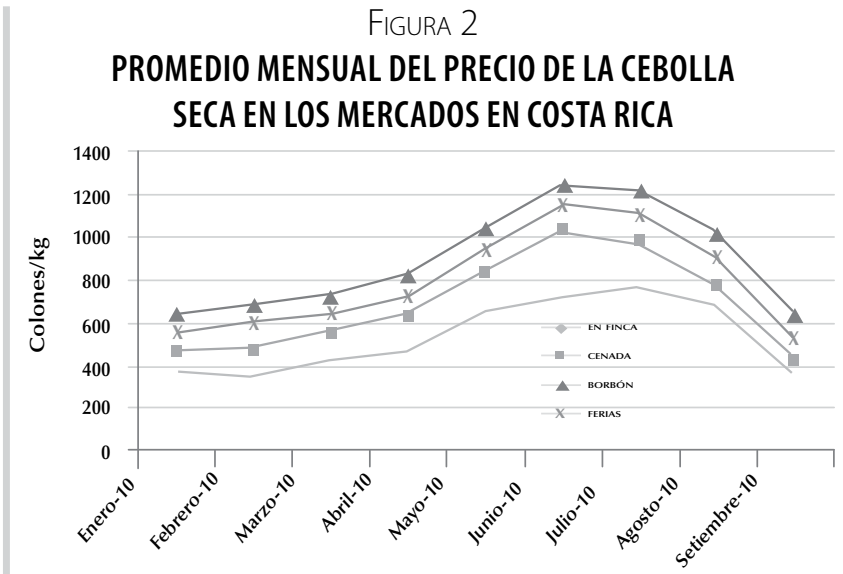

Fuente: Consejo Nacional de Producción (2010), servicio de información e inteligencia de mercados tomado el 09 de octubre de 2010.

\section{Análisis del requerimiento de agua para incre- mentar la producción de cebolla con el SCALL o Reservorio en un Ciclo en la época seca}

Los requerimientos que se analizaron y realizaron para implementar el SCALL con el cultivo de cebolla de detallan en la Tabla 8.

\section{CULTIVO CEBOLLA Y SUS REQUERIMIENTOS PARA LA UTILIZACIÓN DEL RESERVORIO EN 240 M²}

\begin{tabular}{|c|c|c|}
\hline $\begin{array}{l}\text { Necesidades de agua del cultivo de Cebolla } \\
\text { Evapotranspiración }=E T 0: 7 \mathrm{~mm} \text {. } \\
\text { Ciclo vegetativo }=105 \text { días. } \\
\text { Uso consecutivo }(\mathrm{Uc})=1 \times 7 \times 105=735 \mathrm{~mm} . \\
\text { Necesidad total de agua para el cultivo en } 240 \mathrm{~m}^{2}=0,735 \times 240 \mathrm{~m}^{2}= \\
\mathbf{1 7 6 , 4} \mathbf{m} \mathbf{3}=176400 \text { litros }\end{array}$ & $\begin{array}{l}\text { Evaporación del reservorio en } 3 \text { meses } \\
\text { Vevap }=10^{*} S^{*} E \\
\text { Vevap }=10^{*}(0.0224)^{*}(240)^{*} 3=161,28 \mathrm{~m}^{3}\end{array}$ & $\begin{array}{l}\text { Dimensiones del reservorio } \\
\text { Largo }{ }^{*} \text { ancho }{ }^{*} \text { profundidad. } \\
14^{*} 16^{*} 1,51=340 \mathrm{~m}^{3}\end{array}$ \\
\hline \multicolumn{3}{|c|}{ Necesidad de agua del cultivo + evaporación del reservorio = volumen necesario de agua para la siembra de cebolla en $240 \mathrm{~m}^{2}$} \\
\hline \multicolumn{3}{|c|}{$176,4+161,28=337,68 \mathrm{~m} 3$} \\
\hline
\end{tabular}

Fuente: Salinas ( 2009). 
Interpretación

Volumen necesario para la siembra de cebolla es $337,68 \mathrm{~m}^{3}<340 \mathrm{~m}^{3}$ volumen almacenado en el reservorio. Por lo tanto, con el reservorio en estudio se puede sembrar $240 \mathrm{~m}^{2}$ de Cebolla.

\section{Ingresos y Costos de Producción}

Los supuestos de ingresos y costos de este proyecto en materia de producción de hortalizas son calculados con base en los datos de la Asociación de agricultores Orgánicos de Cerro Verde quienes tienen más de 15 años de experiencia en producción y comercialización de productos orgánicos, además de información de ferias del agricultor por el Consejo Nacional de Producción (CNP, figura 2), Centro Nacional de Abastecimiento y Distribución de Alimentos (CENADA) y los precios por región.

Los supuestos de ingresos por ventas y costos de producción en peces (Tilapias) fueron calculados con base en información de la Universidad Técnica Nacional y el Instituto Costarricense de Pesca y Acuicultura, tanto los costos como los ingresos se reportaron en dólares (USD).

\section{Vida Útil de los activos}

Se estima una vida útil del reservorio de 20 años y del sistema de riego de 5 años, ambos son depreciados con el medo de línea recta el valor de rescate final se estimó en un 10\% del valor inicial de cada uno de ellos.

\section{TABLA 9}

\section{CÁLCULO DE LA DEPRECIACIÓN DEL SISTEMA}

\begin{tabular}{|l|c|c|c|}
\hline \multicolumn{1}{|c|}{ Activos } & USD & Cantidad & $\begin{array}{c}\text { Depreciación de } \\
\text { línea recta en 1 año }\end{array}$ \\
\hline Reservorio & 4210,44 & $345 \mathrm{~m}^{3}$ & 210,522 \\
\hline Sistema de riego & 550 & $1000 \mathrm{~m}^{2}$ & 27,5 \\
\hline $\begin{array}{l}\text { Total de depreciación } \\
\text { de activo }\end{array}$ & & & 238,022 \\
\hline
\end{tabular}

Fuente: elaboración propia.
En la Tabla 10 se muestran los costos anuales, tanto fijos como variables, en la producción de ambos cultivos cebolla y tilapia.

\section{TABLA 10 \\ COSTOS ANUALES DE PRODUCCIÓN DE CEBOLLA Y TILAPIA EN USD}

\begin{tabular}{|l|c|c|}
\hline \multicolumn{1}{|c|}{ Costos Fijos } & $\begin{array}{c}\text { Cantidades } \\
\text { USD }\end{array}$ & $\begin{array}{c}\text { Proporción } \\
\text { porcentual } \%\end{array}$ \\
\hline Materiales y suministros & $\$ 100,00$ & 1,40 \\
\hline Depreciación y amortización de activos & $\$ 238,00$ & 3,34 \\
\hline Teléfono & $\$ 100,00$ & 1,40 \\
\hline Imprevistos (5\%) & $\$ 21,90$ & 0,31 \\
\hline Total de costos fijos & $\$ 459,90$ & 6,45 \\
\hline Costos Variables & & \\
\hline Alevines & $\$ 170,00$ & 2,38 \\
\hline Semilla & $\$ 200,00$ & 2,81 \\
\hline Alimento de tilapia. & $\$ 3100,00$ & 43,48 \\
\hline Mano de obra directa & $\$ 2600,00$ & 36,47 \\
\hline Preparación del sustrato & $\$ 100,00$ & 1,40 \\
\hline Envasaje, embalaje y etiqueta & $\$ 500,00$ & 7,01 \\
\hline Imprevistos (5\%) & $\$ 333,50$ & 4,68 \\
\hline Total de costos Variables & $\$ 6670,00$ & 93,55 \\
\hline Total de costos operativos & $\$ 7129,90$ & 100,00 \\
\hline
\end{tabular}

Fuente: elaboración propia.

\section{Ingresos por la producción de Cebolla}

En la tabla 11 se presentan tres diferentes escenarios sobre costo, rendimiento e ingresos brutos para la siembra de $240 \mathrm{~m}^{2}$ de cebolla en Cerro Verde de Nicoya, con el SCALL o reservorio. Se muestra cómocomo incrementa un ciclo de producción, dando mayores rendimientos durante la época seca por factores relacionados con mayor luz en el cultivo. 
TABLA 11

\section{INGRESOS DE LA PRODUCCIÓN DE CEBOLLA EN 3 POSIBLES ESCENARIOS: PESIMISTA, NORMAL Y OPTIMISTA}

\begin{tabular}{|c|c|c|c|c|c|c|c|c|c|}
\hline Cultivo & $\begin{array}{c}\text { Ciclos } \\
\text { del cultivo } \\
\text { (días) }\end{array}$ & $\begin{array}{c}\text { Ciclos } \\
\text { posibles } \\
\text { al año }\end{array}$ & $\begin{array}{c}\text { Número } \\
\text { de Ciclo } \\
\text { sin riego }\end{array}$ & $\begin{array}{c}\text { Costos } \\
\text { por ciclo }\end{array}$ & $\begin{array}{c}\text { Costos } \\
\text { por año } \\
\text { sin riego }\end{array}$ & $\begin{array}{c}\text { Costos } \\
\text { por año } \\
\text { con riego }\end{array}$ & $\begin{array}{c}\text { Rendimiento } \\
\text { ler Ciclo en kg } \\
\text { (sin riego) }\end{array}$ & $\begin{array}{c}\text { Rendimiento } \\
\text { 2do Ciclo en kg } \\
(\text { sin riego) }\end{array}$ & $\begin{array}{c}\text { Rendimiento } \\
\text { 3er Ciclo en kg } \\
\text { (con riego) }\end{array}$ \\
\hline Cebolla & 105 & 3 & 2 & 219,43 & 438,86 & 790,29 & 960 & 960 \\
\hline
\end{tabular}

\begin{tabular}{|l|c|c|}
\hline \multicolumn{1}{|c|}{ Escenarios posibles } & Precio de venta USD $\$$ & Ingresos brutos \\
\hline Escenario pesimista en precio de venta & 1,18 & 3681,6 \\
\hline Escenario normal en precio de venta & 1,67 & 5210,4 \\
\hline Escenario optimista en precio de venta & 1,86 & 5803,2 \\
\hline
\end{tabular}

Fuente: Elaboración propia con base a la información del CNP (2010), servicio de información e inteligencia de mercados.

\section{Ingresos por la producción de tilapia}

En la tabla 12 se presentan tres diferentes escenarios sobre costo, rendimiento e ingresos brutos para la producción de 2000 alevines (tilapia pequeña) con el SCALL o Reservorio instalado en Cerro Verde de Nicoya.

TABLA 12

\section{INGRESOS DE LA PRODUCCIÓN DE TILAPIA EN 3 POSIBLES ESCENARIOS: PESIMISTA, NORMAL Y OPTIMISTA.}

\begin{tabular}{|c|c|c|c|}
\hline Cantidad (alevines) & Rendimiento de Producción kg & Costo de producción por kg en USD & Costo anual de producción USD \\
\hline 2000 & 1700 & 2,15 & 3655 \\
\hline
\end{tabular}

\begin{tabular}{|l|c|c|}
\hline \multicolumn{1}{|c|}{ Escenarios posibles en precio de venta } & Precio USD & Ventas USD \\
\hline Escenario pesimista & 3,93 & 6681 \\
\hline Escenario normal & 4,9 & 8330 \\
\hline Escenario 0ptimista & 5,88 & 9996 \\
\hline
\end{tabular}

Fuente: Elaboración propia basado en información suministrada por la Universidad Técnica de Costa Rica y el Instituto Costarricense de Pesca y Acuicultura.

En la tabla 13 se muestra el flujo de efectivo para el escenario normal de ambos productos (tilapia y cebolla) como un todo: ingresos anuales de producción versus costos totales. 
TABLA 13

FLUJO NETO DE EFECTIVO SIN FINANCIAMIENTO BANCARIO

\begin{tabular}{|c|c|c|c|c|c|c|}
\hline Cuentas & Año 0 & Año 1 & Año 2 & Año 3 & Año 4 & Año 5 \\
\hline Ingresos & & $\$ 13540,40$ & $\$ 13540,40$ & $\$ 13540,40$ & $\$ 13540,40$ & $\$ 13540,40$ \\
\hline Costoscostos fijos & & $\$ 459,90$ & $\$ 459,90$ & $\$ 459,90$ & $\$ 459,90$ & $\$ 459,90$ \\
\hline Costoscostos variables & & $\$ 7129,90$ & $\$ 7129,90$ & $\$ 7129,90$ & $\$ 7129,90$ & $\$ 7129,90$ \\
\hline Menosmenos depreciación de activo & & $\$ 238,02$ & $\$ 238,02$ & $\$ 238,02$ & $\$ 238,02$ & $\$ 238,02$ \\
\hline Utilidadutilidad antes de impuestos de renta & & $\$ 5712,58$ & $\$ 5712,58$ & $\$ 5712,58$ & $\$ 5712,58$ & $\$ 5712,58$ \\
\hline Impuesto de renta (10\%) & & $\$ 23,80$ & $\$ 23,80$ & $\$ 23,80$ & $\$ 23,80$ & $\$ 23,80$ \\
\hline Utilidadutilidad neta & & $\$ 5688,78$ & $\$ 5688,78$ & $\$ 5688,78$ & $\$ 5688,78$ & $\$ 5688,78$ \\
\hline Másmas depreciación y amortización de activo & & $\$ 238,02$ & $\$ 238,02$ & $\$ 238,02$ & $\$ 238,02$ & $\$ 238,02$ \\
\hline Flujoflujo operativo neto de efectivo & $-\$ 7499,32$ & $\$ 5926,80$ & $\$ 5926,80$ & $\$ 5926,80$ & $\$ 5926,80$ & $\$ 5926,80$ \\
\hline \multicolumn{7}{|l|}{ Inversiones Inversiones } \\
\hline Infraestructura & $\$ 4670,00$ & & & & & \\
\hline Equipo de producción & $\$ 800,00$ & & & & & \\
\hline Equipo de oficina & $\$ 200,00$ & & & & & \\
\hline Capital de trabajo & $\$ 939,32$ & & & & & \\
\hline Activo nominal & $\$ 1000,00$ & & & & & \\
\hline \multicolumn{7}{|l|}{ Parámetros financieros } \\
\hline Tasa Interna de Retorno (TIR) & $74,09 \%$ & & & & & \\
\hline Valor Actual Neto (VAN) & $\$ 16189,63$ & & & & & \\
\hline Tasa de recarga financiera & $7,96 \%$ & & & & & \\
\hline Valor actual neto de los ingresos & $\$ 23688,95$ & & & & & \\
\hline Valor actual neto de los egresos & $\$ 7499,32$ & & & & & \\
\hline Razón Beneficio costo & $\$ 3,16$ & & & & & \\
\hline
\end{tabular}

Fuente: elaboración propia.

1163 (1), Enero - Junio, 2012 


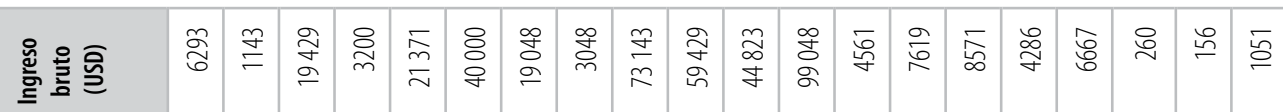

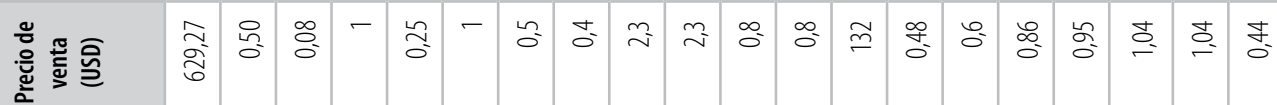

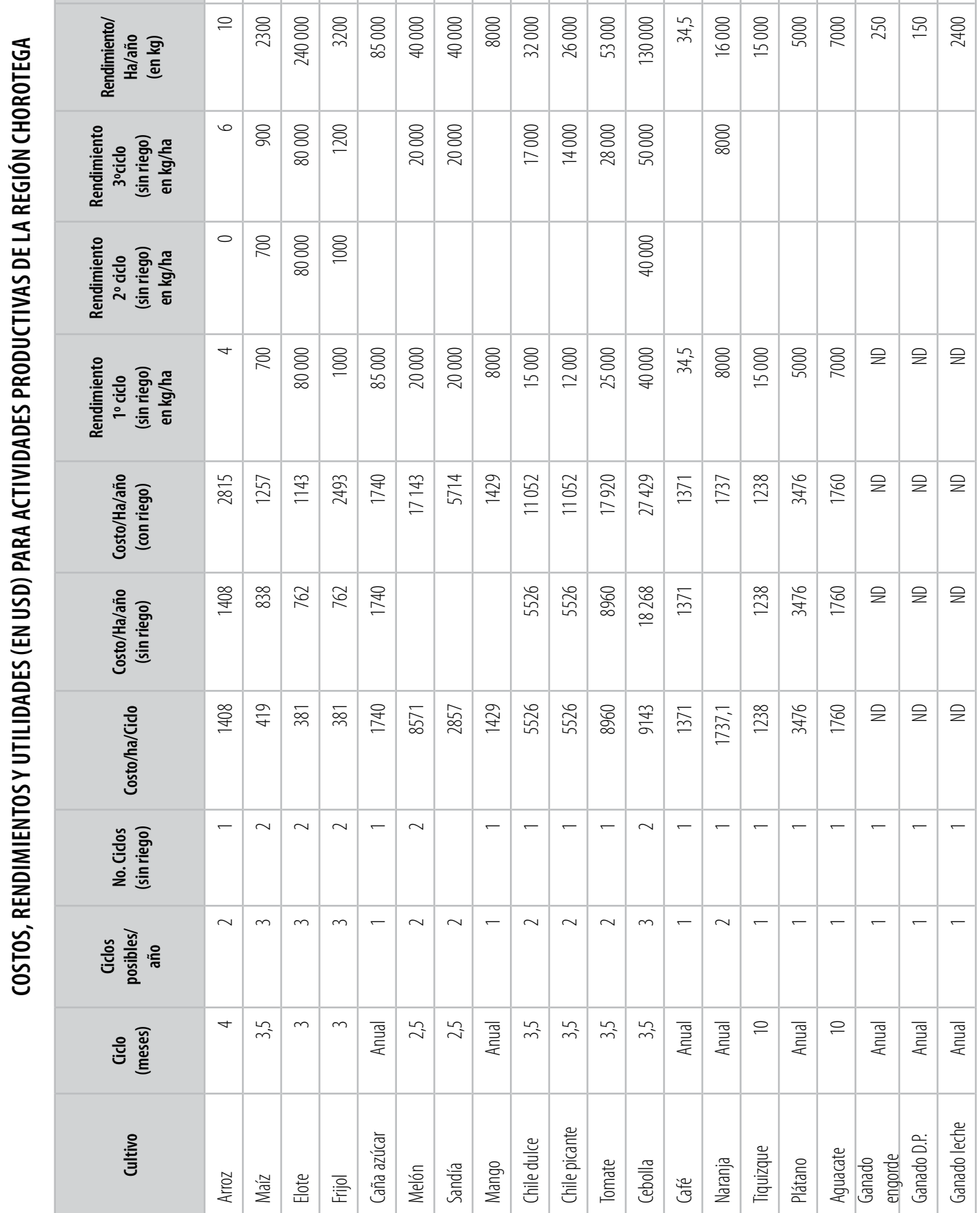




\section{RESULTADOS Y DISCUSIÓN}

\section{Análisis de indicadores financieros}

sin financiamiento bancario

La evaluación financiera de esta investigación comprende 3 de los criterios más utilizados para evaluar proyectos de inversión, es decir, nos referimos al Valor Actual Neto (VAN), Tasa Interna de Retorno (TIR) y Razón Beneficio/Costo o Índice de Rentabilidad.

Se utilizó una tasa comparativa en dólares (USD) de la TIR de 1,11\%, así como la tasa de costo de oportunidad de 7,96\% en dólares, basada en información del Banco Central de Costa Rica (2005-2010).

\section{Valor actual neto (VAN)}

El Valor Actual Neto (VAN) de un proyecto mide en dinero corriente el grado de mayor riqueza que tendrá el inversionista en el futuro. Se dice que cuando el VAN es positivo el negocio es viable porque rinde sobre los costos del capital exigido. EI VAN de este proyecto es \$16 189,63.

Tasa interna de retorno

o de rendimiento (TIR)

La tasa interna de retorno (TIR), mide la rentabilidad como un porcentaje y muestra en promedio el porcentaje de rentabilidad que tendrá el proyecto por cada periodo. En otros términos se dice que la TIR corresponde a la tasa de interés que torna cero el VAN de un proyecto, anulándose la rentabilidad del mismo. De esta forma se puede conocer hasta qué nivel puede crecer la tasa de descuento y aún el proyecto sigue siendo rentable financieramente. La TIR de este proyecto es $\mathbf{7 4 , 0 9 \%}$.
Razón Beneficio/Costo

La Razón Beneficio/Costo compara el valor actual de los ingresos con el valor actual de los egresos: Para este proyecto la razón beneficio/ costo es de \$3,16 lo que significa que por cada dólar invertido, el proyecto genera un valor presente $\$ 3,16$ considerando una tasa de costo de oportunidad de 7,96\%.

Su fórmula se expresa: VAN ingresos $\div$ VAN de egresos

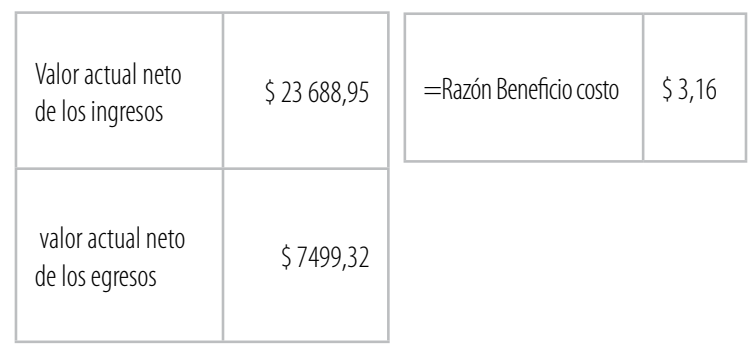

Análisis de indicadores financieros con financiamiento bancario

En la tabla 15 se muestra un escenario con financiamiento bancario a 5 años plazo. De un crédito que cubra la inversión total del proyecto por una suma de $\$ 7500$ USD necesarios para implementar el SCALL o reservorio a una tasa de 7,75\% anual mostrando parámetros financieros positivos para tres razones financieras analizadas: valor actual neto, tasa interna de retorno y razón costo beneficio. 
TABLA 15

ANÁLISIS DE LOS PARÁMETROS FINANCIEROS CON FINANCIAMIENTO BANCARIO A 5 AÑOS DE \$7500 USD AL 7,75\% ANUAL

\begin{tabular}{|l|r|r|r|r|r|r|}
\hline & \multicolumn{1}{|c|}{ año 0} & \multicolumn{1}{|c|}{ año 1 } & \multicolumn{1}{|c|}{ año 2 } & año 3 & año 4 & año 5 \\
\hline Crédito Bancario & $\$ 7500,00$ & & & & & \\
\hline Pago de amortizaciónAmortización más intereses & & $\$ 1814,13$ & $\$ 1814,13$ & $\$ 1814,13$ & $\$ 1814,13$ & $\$ 1814,13$ \\
\hline Flujoflujo operativo neto de efectivo & $-\$ 7499,32$ & $\$ 4112,67$ & $\$ 4112,67$ & $\$ 4112,67$ & $\$ 4112,67$ & $\$ 4112,67$ \\
\hline Parámetros financieros & & & & & & \\
\hline Tasa Interna de Retorno (TIR) & $46,80 \%$ & & & & & \\
\hline Valor Actual Neto (VAN) & $\$ 8938,70$ & & & & & \\
\hline Tasa de recarga financiera & $7,96 \%$ & & & & & \\
\hline Valor actual neto de los ingresos & $\$ 16438,02$ & & & & & \\
\hline Valor actual neto de los egresos & $\$ 7499,32$ & & & & & \\
\hline Razón Beneficio costo & $\$ 2,19$ & & & & & \\
\hline
\end{tabular}

\section{CONCLUSIONES}

En Guanacaste el déficit del recurso hídrico en la época seca genera pérdidas millonarias en el sector agropecuario contribuyendo al aumento de la pobreza y el desempleo en la región.Región. La investigación demostró que un SCALL o reservorio puede ser utilizado con fines productivos en la época seca y en la época lluviosa.

Los sistemas de captación de agua lluvia se plantean como una alternativa de producción tecnológicamente no compleja y ecológicamente viable y desde el punto de vista financiero se concluye que la instalación de un sistema de captación de agua lluvia para ser utilizado en la producción de tilapias y hortalizas es una alternativa muy rentable con o sin financiamiento, que desarrollado a gran escala permitiría disminuir las pérdidas en agricultura que enfrenta año con año la provincia guanacasteca durante la época de seca. Con, con la investigación se identificaron criterios técnicos y humanos necesarios para la producción, los materiales necesarios para la instalación de las infraestructura son de fácil acceso a nivel nacional, se concluye que es importante revestir el reservorio o SCALL con geomembrana, pues a pesar de que su costo es relativamente elevado, al ser un material de excelente calidad proporciona una vida útil de la estructura de 25 años asegurando la inversión.

\section{REFERENCIAS}

Anaya, M. (1994). Sistemas de captación de agua de lluvia para uso doméstico en América Latina y el Caribe, Manual Técnico. México: IICA.

Anaya, M y Martínez, J. (2007). Manual de sistemas de captación y aprovechamiento del agua de lluvia para uso doméstico y consumo humano en América Latina y el Caribe. México: IICA.

Cubero D. (1996). Manual de Conservación de suelos y aguas. Ministerio de Agricultura y Ganadería. Costa Rica: EUNED.

Herrera, W. (1986). Clima de Costa Rica en Vegetación y Clima de Costa Rica. Costa Rica: EUNED.

Lawrence, J. Gitman, A. (2009) Principios de administración financiera 2. USA: Person Education. 
RNA Revista Nacional de Administración

National Academy of Sciences. (1974). More water for arid lands. Promising Technologies and Research Opportunities. Washington, DC. USA: NAS.

Ralph, S. y Polimeni, P. D. (1990). Contabilidad de costos Conceptos y aplicaciones para la toma de decisiones gerenciales. México: McGRAW-HILL.

Consejo Nacional de Producción (2010). Servicio de información e inteligencia de mercado, precio de papa y ceboIla. Recuperado el 09 de octubre de 2010, de: http:// www.cnp.go.cr/index.php?idS=1 yidM=79

Valverde, J. (2007). Riego y Drenaje. San José, Costa Rica: Editorial Universidad Estatal a Distancia (UNED).

Salinas, A (2009). Manual de especificaciones técnicas-básicas para la elaboración de estructuras de captación de agua de lluvia (SCALL) en el sector agropecuario de Costa Rica y recomendaciones para su utilización. Universidad Nacional: CEMEDE.

Recibido: 31-01-2011

Aceptado: 08-02-2012 